\title{
Methyl group, hyperconjugation and human diseases
}

Yuchuan Wang ${ }^{2}$, Lipeng Liao ${ }^{1}$, Yuhan Lin ${ }^{1}$, Chanyuk Lam ${ }^{1}$, Jess Ouyang Lan ${ }^{1}$, Min Fu³, Qiuyun Liu, ${ }^{1}$,

${ }^{1}$ School of Life Sciences, Sun Yat-Sen University, Guangzhou 510275, China.

${ }^{2}$ Center for Synthetic Biology Engineering Research, Shenzhen Institutes of Advanced Technology, Chinese Academy of Sciences, Shenzhen, Guangdong 518055, China.

${ }^{3}$ Deyang Health Committee, Sichuan, China

*Correspondence author: Qiuyun Liu, School of Life Sciences, Sun Yat-sen University, Guangzhou 510275, China.

E-mail address: 1sslqy@mail.sysu.edu.cn (Q. Liu) 


\begin{abstract}
Methylations are widespread in the biological kingdom. However, their chemical and biochemical roles have not been fully dissected. A hypothesis was put forward previously in which methylation is proposed to lead to hyperconjugation, whose electron donating feature weakens the chemical bonds and increases the bond lengths of $\mathrm{C}-\mathrm{N}$, therefore resulting in stronger secondary bonding between nitrogen atoms and cations or protonation of nitrogen atoms. Consequently ion homeostasis of the cells is compromised giving rise to certain diseases. Protonation of nitrogen atoms in 5-methyl cytosine enhances interaction with negatively charged phosphate groups and contributes to the formation of heterochromatin.
\end{abstract}

\title{
Keywords
}

methylation; hyperconjugation; electron delocalization, human diseases 
DNA methylation has been linked to cancer, atherosclerosis and aging.[1-3] Histone methylation is also related to aging, neurodegenerative illnesses, and intellectual debility such as Rubinstein-Taybi Syndrome and X-linked mental retardation. [4-8]

DNA methylation mostly arises at the carbon-5 position of a cytosine base. It underlies transcriptional repression, and is a fundamental feature of heterochromatin [9]. CpG islands typically appear in gene promoters. They are often devoid of methylation allowing gene activation [10-13]. Methylation enables hyperconjugation with adjacent carbon atoms, and the higher electron density in $\mathrm{C}^{4}$ indicated by NMR data in comparison to cytosine extends $\mathrm{C}^{4}-\mathrm{N}$ bond length [14-16], allowing easier protonation in the nitrogen atom and generation of a positive charge.[16-18] The electrostatic interactions between charged amino groups and negatively charged phosphate groups generate compact DNA and contribute to the formation of heterochromatin, consequently repressing gene expression. The protonation in the amino nitrogen of 5-methyl cytosine can hasten $\mathrm{C}$ to $\mathrm{T}$ transition mutations, accelerating evolution and speciation.

Methylation of lysine 9 of histone $\mathrm{H} 3$ has been associated with constitutively transcriptionally silent chromatin. Yet, tri-methylation of histone H3 at lysine 4 is strongly linked with transcriptional activation. Tri-methylation here would bring about a positive charge on the tail. However, the electron delocalization of 3 electron-donating methyl groups can effectively reduce the positive charge and stabilize lysine 4 of histone $\mathrm{H} 3$.

\section{Conflict of interest}

None declared.

\section{ACKNOWLEDGMENT}

This work was supported by the Science and Technology Transformation Program of Sun Yat-sen University of China (33000-18843234) and Guangzhou Science and Technology Program (201804010328), Guangdong Natural Science Foundation (S2011010004264) and Guangdong Science and Technology Program (No. 2008B020100001) to Q. Liu; National Science Foundation of China (21601209) to Y. Wang. We appreciate discussions and suggestions from Weiguo Cao and Jiaming Zhang.

\section{REFERENCES}

[1] Zhang FF, Cardarelli R, Carroll J, Zhang S, Fulda KG, Gonzalez K, et al. Physical activity and global genomic DNA methylation in a cancer-free population. Epigenet 2011;6 (3): 293-299.

[2] Lund GL, Andersson L, Lauria M, Lindholm M, Fraga MF, Villar-Garea A, et al. DNA methylation polymorphisms precede any histological sign of atherosclerosis in mice lacking Apolipoprotein E.. J Biol Chem 2004;279 (28): 29147-54.

[3] Gonzalo S. Epigenetic alterations in aging. J Appl Physiol 2010;109 (2):586-597.

[4] Kramer JM. Epigenetic regulation of memory: implications in human cognitive disorders. Biomol Concepts 2013;4 (1): 1-12. 
[5] Tobias L, Heldring N, Hermanson O. Like a rolling histone: Epigenetic regulation of neural stem cells and brain development by factors controlling histone acetylation and methylation. Biochim Biophys Acta - Gen Subj 2013;1830 (2): 2354-60.

[6] Ho E, Beaver LM, Williams DE, Dashwood RH. Dietary Factors and Epigenetic Regulation for Prostate Cancer Prevention. Adv Nutrition 2011; 2(6): 497-510

[7] Greer EL, Shi Y. Histone methylation: a dynamic mark in health, disease and inheritance. Nat Rev Genet 2014;13(5): 343-57.

[8] Zhang Y, Reinberg D. Transcription regulation by histone methylation: interplay between different covalent modifications of the core histone tails. Genes Dev 2001; 15(18): 2143-60.

[9] Miller OJ, Schnedl W, Allen J, Erlanger BF. 5-Methylcytosine localised in mammalian constitutive heterochromatin. Nature 1974;251:636-7

[10] Bird A. DNA methylation de novo. Science 1999;286:2287-8

[11] Bird AP. CpG-rich islands and the function of DNA methylation. Nature 1986;321:209-13

[12] Jaenisch R, Bird A. Epigenetic regulation of gene expression: how the genome integrates intrinsic and environmental signals. Nat Genet 2003;33(Suppl):245-54

[13] Gardiner-Garden M, Frommer M. CpG islands in vertebrate genomes. J Mol Biol 1987;196:261-82

[14] http://mmcd.nmrfam.wisc.edu/test/cqsearch.py?cqid=cq 01488

[15] http://mmcd.nmrfam.wisc.edu/test/cqsearch.py?cqid=cq_00273

[16] Wang K, Yang X, Yu W, Li Z, Liang, Y, Liu Q, A chemical perspective for DNA methylation, Science 2017; http://science.sciencemag.org/content/354/6314/843.6/tab-e-letters

[17] Wang Y, Wang Z, Cui, L, Yan S, Liu Q. Why some circulating DNA methylation markers can be used for diagnosis and prognosis of cancers. Science 2018, http://science.sciencemag.org/content/354/6314/843.6/tab-e-letters

[18] An S, Zhang J, Wang Y, Zhang Y, Liu Q. The roles of $\mathrm{N}^{6}$-Methyladenosine in human diseases. Biochem Insights 2019;12:1178626419883248. https://doi.org/10.1177/117862641988324 


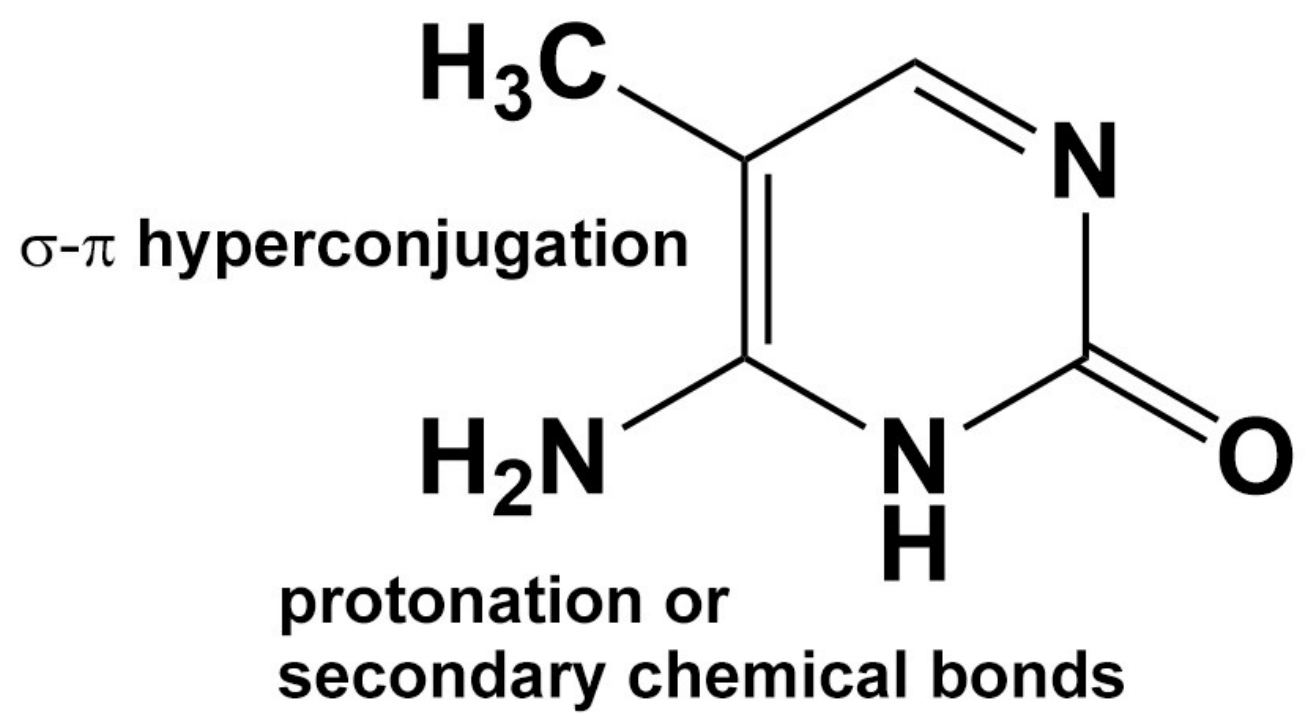

Fig 1. Chemical perspective of cytosine methylation. 